\title{
SINGULAR PERTURBATION METHODS FOR A CLASS OF INITIAL AND BOUNDARY VALUE PROBLEMS IN MULTI-PARAMETER CLASSICAL DIGITAL CONTROL SYSTEMS
}

\author{
M. S. KRISHNARAYALU ${ }^{1}$
}

(Received 1 August, 2002; revised 9 January, 2003)

\begin{abstract}
A stable linear time-invariant classical digital control system with several widely different small coefficients multiplying the lowest functions is considered. It is formulated as a multi-parameter singularly perturbed system. Perturbation methods are developed for both initial and boundary value problems based on asymptotic expansions of the perturbation parameters. The approximate solution consists of an outer solution and a number of boundary layer correction solutions equal to the number of initial conditions lost in the process of degeneration. An example is provided for illustration.
\end{abstract}

\section{Introduction}

The dynamics of many continuous-time and digital systems are described by high order differential and difference equations, respectively. Frequently, the presence of small parameters such as time constants, masses, moments of inertia, inductances and capacitances is the source of increased order in the system. A system in which the suppression of a small parameter is responsible for the degeneration of the dimension of the system is called a singularly perturbed system. Such a system possesses widely separated clusters of eigenvalues exhibiting slow and fast phenomena or time-scale phenomena. The high dimensionality coupled with the time-scale behaviour makes the system computationally stiff resulting in the use of extensive numerical routines.

We frequently encounter boundary value problems (BVPs) in optimal control [17]. The solution of BVPs is always a concern. The solution of two-point boundary value problems (TPBVPs) of stiff systems requires special methods such as shooting techniques [16]. Even these special methods are trial and error methods. The singular perturbation methods, which are not trial and error methods, remove stiffness, reduce

${ }^{1} 1 / 19$ Meadow Crescent, Meadowbank, NSW 2114, Australia; e-mail: sreemovva@hotmail.com.

(C) Australian Mathematical Society 2004, Serial-fee code 1446-1811/04 
the order of the system and satisfy the specified boundary conditions of the system. The crux of singular perturbation theory is as follows. The degenerate system, obtained by suppressing the perturbation (small) parameters is of reduced order and can satisfy the specified boundary conditions of the slow modes only. The rest of the specified boundary conditions of the fast modes are lost in the process of stiffness removal (degeneration). Boundary layers are formed due to the nonuniform convergence of the exact solution to the degenerate solution. Boundary layers correspond to the rapid region of transition in the exact solution. Now boundary layer corrections have to be added to recover the lost boundary conditions and to improve the degenerate solution. Also boundary layer corrections should ensure that the solution is unique.

Singular perturbation theory in continuous-time control systems is well documented and has reached a level of maturity $[1,4,11,14,18]$. Singular perturbation analysis of digital systems is gaining momentum $[2,3,5-13,15,19]$. Research into singular perturbation analysis of digital systems started with one small parameter (two-timescales) $[12,15]$ and then extended to two small parameters (three-time-scales) $[9,10]$. These singular perturbation methods were applied to optimal control problems $[2,7,8]$. Now they are being extended to multi-parameters with multi-time-scales. Multi-timescale problems are prevalent in engineering and other applications [11]. Already singular perturbation methods, for initial and boundary value problems of multiparameter multi-time-scale linear time-invariant (LTI) digital control systems with stable fast modes giving rise to boundary layers at the initial point $(k=0)$, are being reported in state space form [6]. In the present paper, we consider the same system in classical form and formulate it as a multi-parameter system and develop perturbation methods for initial and boundary value problems.

\section{Statement of the problem}

We consider an $\mathfrak{N}(=m+n+p+q+\cdots+s)$ th-order stable linear time-invariant digital control system described by a difference equation as

$$
\sum_{i=\mathfrak{N}}^{0} d_{i} w(k+i)=d u(k),
$$

where the coefficients $d_{i}$ form distinct groups based on the order of magnitude:

$$
\begin{aligned}
O\left(d_{i}\right), \quad i=0, \ldots, s-1, \\
\\
\ll O\left(d_{i}\right), \quad i=s, \ldots, \mathfrak{N}-m-n-p-1, \\
\quad \ll O\left(d_{i}\right), \quad i=\mathfrak{N}-m-n-p, \ldots, \mathfrak{N}-m-n-1, \\
\ll O\left(d_{i}\right), \quad i=\mathfrak{N}-m-n, \ldots, \mathfrak{N}-m-1, \\
\ll O\left(d_{i}\right), \quad i=\mathfrak{N}-m, \ldots, \mathfrak{N} .
\end{aligned}
$$


In other words, the system is a multi-time-scale one with clusters of eigenvalues of different orders of magnitude giving rise to slow, fast, faster and fastest modes. The basic idea of one- and two-parameter problems and the relationship between the coefficients and eigenvalues is explained in the Appendix where a second-order system is considered.

Based on the two-parameter problem [10, Appendix] and multi-parameter problem [6], by a suitable choice of coefficients, (2.1) may be written as

$$
\begin{aligned}
w(k+\mathfrak{N}) & +a_{\mathfrak{N}-1} w(k+\mathfrak{N}-1)+\cdots+a_{\mathfrak{N}-m} w(k+\mathfrak{N}-m) \\
& +a_{\mathfrak{N}-m-1} h_{1} w(k+\mathfrak{N}-m-1) \\
& +\cdots+a_{\mathfrak{N}-m-n} h_{1}^{n} w(k+\mathfrak{N}-m-n) \\
& +a_{\mathfrak{N}-m-n-1} h_{1}^{n+1} h_{2} w(k+\mathfrak{N}-m-n-1) \\
& +\cdots+a_{\mathfrak{N}-m-n-p} h_{1}^{n+p} h_{2}^{p} w(k+\mathfrak{N}-m-n-p) \\
& +a_{\mathfrak{N}-m-n-p-1} h_{1}^{n+p+1} h_{2}^{p+1} w(k+\mathfrak{N}-m-n-p-1) \\
& +\cdots+a_{0} h_{1}^{\mathfrak{N}-m} h_{2}^{\mathfrak{N}-m-n} h_{3}^{\mathfrak{N}-m-n-p} \cdots h_{f}^{s} w(k)=b u(k),
\end{aligned}
$$

where $h_{1}, h_{2}, h_{3}, \ldots, h_{f}$ are the interrelated perturbation parameters corresponding to the groups of coefficients which are smaller in magnitude and $b=d / d_{\mathfrak{N}}$. These perturbation parameters approach zero simultaneously.

If the boundary conditions of the system (2.2) are

$$
w(j)=w(k=j), \quad j=0,1,2, \ldots, \mathfrak{N}-1,
$$

where $w(k=j)$ are given values, then the problem at hand is an initial value problem (IVP).

If the boundary conditions of the system (2.2) are given as

$$
w(j)=w(k=j), \quad j=0,1,2, \ldots, \mathfrak{N}-m-1
$$

and

$$
w(N-i)=w(k=N-i), \quad i=m-1, m-2, \ldots, 1,0,
$$

then we have a BVP, where $N$ is a fixed integer indicating the final time.

The degenerate system corresponds to slow eigenvalues ignoring the fast groups of eigenvalues. The degenerate system, obtained by suppressing the perturbation parameters in (2.2) simultaneously, is given by

$$
w^{0 \ldots 0}(k+\mathfrak{N})+a_{\mathfrak{N}-1} w^{0 \ldots 0}(k+\mathfrak{N}-1)+\cdots+a_{\mathfrak{N}-m} w^{0 \ldots 0}(k+\mathfrak{N}-m)=b u(k) .
$$

Equation (2.5) is of order $m$ and naturally can satisfy $m$ initial conditions (corresponding to the slow modes) $w(j), j=\mathfrak{N}-m, \mathfrak{N}-m+1, \ldots, \mathfrak{N}-1$, in the case of an 
IVP or boundary conditions (2.4b) in the case of a BVP. The remaining $(\mathfrak{N}-m)$ initial conditions (2.4a) (corresponding to fast modes) are lost in the process of degeneration and $(\mathfrak{N}-m)$ boundary layers are formed. Hence the above IVP and BVP are said to be in singularly perturbed form. These $(\mathfrak{N}-m)$ initial conditions are recovered by the following perturbation method where the approximate solution consists of an outer solution (solutions outside the boundary layers) and the number of boundary layer correction solutions (solution inside the boundary layers) equals the number of initial conditions lost in the process of degeneration. The external input $u(k)$ is independent of the perturbation parameters and will not be affected by their suppression.

\section{Singular perturbation method}

3.1. Outer solution We assume asymptotic expansions in the perturbation parameters for the outer solution as

$$
w_{0}(k)=\sum_{i, j, \ldots, r \geq 0}^{g} w^{i j \ldots r}(k) h_{1}^{i} h_{2}^{j} \cdots h_{f}^{r},
$$

where $g$ is the desired order of approximation. Substituting (3.1) into (2.2) and equating the coefficients of like powers of the perturbation parameters, a set of equations will be obtained. For the zeroth-order approximation $\left(h_{1}^{0} h_{2}^{0} \cdots h_{f}^{0}\right)$, the resulting equation is the same as that given by (2.5). For the first-order approximation:

$$
\begin{aligned}
& w^{10 \ldots 0}(k+\mathfrak{N})+a_{\mathfrak{N}-1} w^{10 \ldots 0}(k+\mathfrak{N}-1)+\cdots+a_{\mathfrak{N}-m} w^{10 \ldots 0}(k+\mathfrak{N}-m) \\
& \quad+a_{\mathfrak{N}-m-1} w^{0 \ldots 0}(k+\mathfrak{N}-m-1)=0 \\
& w^{\alpha}(k+\mathfrak{N})+a_{\mathfrak{N}-1} w^{\alpha}(k+\mathfrak{N}-1)+\cdots+a_{\mathfrak{N}-m} w^{\alpha}(k+\mathfrak{N}-m) \\
& \quad+a_{\mathfrak{n}-m-1} w^{\alpha}(k+\mathfrak{N}-m-1)=0,
\end{aligned}
$$

where $\alpha=010 \ldots 0, \ldots, 0 \ldots 01$.

3.2. Boundary layer correction (BLC) solutions The transformations to be applied to (2.2), to generate transformed systems corresponding to each perturbation parameter, are

$$
\begin{array}{rlrl}
w_{c}(k) & =w(k) / h_{1}^{k-c}, & c & =\mathfrak{N}-m-1, \ldots, \mathfrak{N}-m-n, \\
w_{d}(k) & =w(k) /\left(h_{1} h_{2}\right)^{k-d}, & d & =\mathfrak{N}-m-n-1, \ldots, \mathfrak{N}-m-n-p, \\
w_{e}(k) & =w(k) /\left(h_{1} h_{2} h_{3}\right)^{k-e}, & e & =\mathfrak{N}-m-n-p-1, \ldots, s, \\
\ldots \ldots \ldots \ldots \ldots \ldots & & \\
w_{v}(k) & =w(k) /\left(h_{1} h_{2} \cdots h_{f}\right)^{k-v}, & v & =s-1, s-2, \ldots, 0 .
\end{array}
$$


We also assume the BLC solutions as asymptotic expansions in the perturbation parameters as

$$
w_{\tau}(k)=\sum_{i, j, \ldots, r \geq 0}^{g} w_{\tau}^{i j \ldots r}(k) h_{1}^{i} h_{2}^{j} \cdots h_{f}^{r}
$$

where $\tau=c, d, e, \ldots, v$ of (3.3).

Substitute (3.4) in the corresponding transformed system and collect the coefficients of like terms of the perturbation parameters. This process gives BLC equations that are to be solved to obtain the total series solution.

3.3. Total series solution The total series solution is given as the sum of the outer series solution (3.1) and the BLC solutions (3.4) as

$$
\begin{aligned}
w^{g}(k)= & \sum_{i, j \ldots, r \geq 0}^{g} w^{i j \ldots r}(k) h_{1}^{i} h_{2}^{j} \cdots h_{f}^{r} \\
& +\sum_{c=n+p+q+\cdots+s-1}^{p+q+\cdots+s} h_{1}^{k-c} \sum_{i, j, \ldots, r \geq 0}^{g} w_{c}^{i j \ldots r}(k) h_{1}^{i} h_{2}^{j} \cdots h_{f}^{r} \\
& +\sum_{d=p+q+\cdots+s-1}^{q+\cdots+s}\left(h_{1} h_{2}\right)^{k-d} \sum_{i, j, \ldots, r \geq 0}^{g} w_{d}^{i j \ldots r}(k) h_{1}^{i} h_{2}^{j} \cdots h_{f}^{r} \\
& +\sum_{e=q+\cdots+s-1}^{g}\left(h_{1} h_{2} h_{3}\right)^{k-e} \sum_{i, j, \ldots, r \geq 0}^{g} w_{e}^{i j \ldots r}(k) h_{1}^{i} h_{2}^{j} \cdots h_{f}^{r} \\
& +\ldots+\sum_{v=s-1}^{0}\left(h_{1} \cdots h_{f}\right)^{k-v} \sum_{i, j, \ldots, r \geq 0}^{g} w_{v}^{i j \ldots r}(k) h_{1}^{i} h_{2}^{j} \cdots h_{f}^{r} .
\end{aligned}
$$

In (3.5) the terms with negative powers for the perturbation parameters of the transformations are defined to be zero.

3.4. Boundary conditions The boundary conditions, required to solve the outer equations (2.5) and (3.2) and the BLC equations resulting from Section 3.2, should be known a priori. These are furnished from the fact that the total series solution (3.5) should satisfy the given boundary conditions. This results in the following relations in the case of an IVP:

$$
\begin{array}{rlrl}
w^{0 \ldots 0}(i) & =w(i), & i & =\mathfrak{N}-1, \ldots, \mathfrak{N}-m, \\
w_{j}^{0 \ldots 0}(j) & =w(j)-w^{0 \ldots 0}(j), & j & =\mathfrak{N}-m-1, \ldots, 1,0, \\
w^{10 \ldots 0}(i) & =0, & i & =\mathfrak{N}-1, \ldots, \mathfrak{N}-m+1, \\
& & w^{10 \ldots 0}(\mathfrak{N}-m)=-w_{\mathfrak{N}-m-1}^{0 \ldots 0}(\mathfrak{N}-m),
\end{array}
$$




$$
\begin{array}{rlrl}
w_{j}^{10 \ldots 0}(j) & =-w^{10 \ldots 0}(j)-w_{j-1}^{0 \ldots 0}(j), & j & =\mathfrak{N}-m-1, \ldots, \mathfrak{N}-m-n+1, \\
w_{i}^{10 \ldots 0}(i) & =-w^{10 \ldots 0}(i), & i & =\mathfrak{N}-m-n, \ldots, 1,0, \\
\ldots \ldots \ldots \ldots \ldots \ldots & i & =\mathfrak{N}-1, \ldots, \mathfrak{N}-m, \\
w^{0 \ldots 01}(i) & =0, & j & =\mathfrak{N}-m-1, \ldots, 1,0 .
\end{array}
$$

Note: In the above equations only one initial condition is specified for each correction equation. The other initial conditions required to solve each correction equation are of zero value.

In the case of a BVP where $N-(m-1)>\mathfrak{N}-m$, the following boundary conditions are to be used in place of (3.6a), (3.6c), (3.6d) and (3.6g) for the outer equations. The initial conditions required for BLC solutions remain the same as given above:

$$
w^{0 \ldots 0}(N-i)=w(N-i), \quad w^{\alpha}(N-i)=0 ;
$$

here $\alpha=10 \ldots 0, \ldots, 0 \ldots 01, i=m-1, \ldots, 1,0$. Furthermore this selection process of boundary conditions ensures that the total series solution (3.5), which consists of the transformations (3.3), is unique.

3.5. Asymptotic correctness In order to prove the asymptotic correctness of the formal series expansions of (3.5), it needs to be shown that

$$
w(k)-w^{g}(k)=O\left(h_{1}^{i} h_{2}^{j} \cdots h_{f}^{r}\right), \quad i+j+\cdots+r=g+1,
$$

where $w(k)$ and $w^{8}(k)$ are the exact and gth-order solutions, respectively. The proof for asymptotic correctness may be obtained in a similar way as in [6].

3.6. Algorithm For a particular order of approximate solution, first find the outer solution. Next, add the BLC corresponding to the least singular transformation. Continuing this process add the BLC corresponding to the most singular transformation finally.

\section{Illustrative example}

Consider the system

$$
\begin{aligned}
w(k+4) & -1.011 w(k+3)+0.1011 w(k+2) \\
& -0.00109 w(k+1)+0.0000009 w(k)=u(k)
\end{aligned}
$$

with boundary conditions $w(0)=12500, w(1)=100, w(2)=10, w(10)=5$; where $u(k)$ is the unit step function. Here the coefficients

$$
0.0000009 \ll 0.00109 \ll 0.1011 \ll 1.011 .
$$


The eigenspectrum of the system $(0.9,0.1,0.01,0.001)$ clearly indicates its multitime-scale nature with stable slow, fast, faster and fastest modes (four-time-scales).

Now (4.1a) may be written in the form of (2.2), with $m=n=p=q=1, s=0$, as

$$
\begin{aligned}
w(k+4) & -1.011 w(k+3)+1.011 h_{1} w(k+2) \\
& -1.3625 h_{1}^{2} h_{2} w(k+1)+1.5625 h_{1}^{3} h_{2}^{2} h_{3} w(k)=u(k),
\end{aligned}
$$

where the perturbation parameters are $h_{1}=0.1, h_{2}=0.08$ and $h_{3}=0.09$.

This problem requires three corrections $w_{c}(k), w_{d}(k)$ and $w_{e}(k)$. Various series solutions are obtained from the total series solution (3.5) as follows.

The degenerate solution (no correction terms) is given by

$$
w(k)=w^{000}(k), \quad 0 \leq k \leq 10
$$

The zeroth-order solution (incorporating correction terms not involving parameter terms) is given by

$$
\begin{array}{ll}
w^{0}(0)=w^{000}(0)+w_{e}^{000}(0), & w^{0}(1)=w^{000}(1)+w_{d}^{000}(1), \\
w^{0}(2)=w^{000}(2)+w_{c}^{000}(2), & w^{0}(k)=w^{000}(k), \quad 3 \leq k \leq 10 .
\end{array}
$$

The first-order solution (incorporating correction terms up to first-order parameter terms) is

$$
\begin{aligned}
w^{1}(0)= & w^{000}(0)+w_{e}^{000}(0)+h_{1} w^{100}(0)+h_{2} w^{010}(0)+h_{3} w^{001}(0) \\
& +h_{1} w_{e}^{100}(0)+h_{2} w_{e}^{010}(0)+h_{3} w_{e}^{001}(0), \\
w^{1}(1)= & w^{000}(1)+w_{d}^{000}(1)+h_{1} w^{100}(1)+h_{2} w^{010}(1)+h_{3} w^{001}(1) \\
& +h_{1} w_{d}^{100}(1)+h_{2} w_{d}^{010}(1)+h_{3} w_{d}^{001}(1) \\
w^{1}(2)= & w^{000}(2)+w_{c}^{000}(2)+h_{1} w^{100}(2)+h_{2} w^{010}(2)+h_{3} w^{001}(2) \\
& +h_{1} w_{c}^{100}(2)+h_{2} w_{c}^{010}(2)+h_{3} w_{c}^{001}(2), \\
w^{1}(k)= & w^{000}(k)+h_{1} w^{100}(k)+h_{2} w^{010}(k)+h_{3} w^{001}(k), \quad 3 \leq k \leq 10 .
\end{aligned}
$$

These series solutions are compared with the exact solution in Table 1.

From Table 1, we note that

(i) The degenerate solution, obtained by making $h_{1}, h_{2}$ and $h_{3}$ equal to zero in (4.1), is unable to satisfy the initial conditions $w(2), w(1)$ and $w(0)$.

(ii) The zeroth-order solution, obtained using (3.5), incorporates BLCs and hence it recovers the initial conditions $w(2), w(1)$ and $w(0)$. Thereafter, that is, for $k>2$, it remains equal to the degenerate solution. 
TABLE 1. Comparison of various series solutions with the exact solution of system (4.1).

\begin{tabular}{|l|l|l|l|l|}
\hline $\boldsymbol{w}(\boldsymbol{k})$ & $\begin{array}{l}\text { Degenerate } \\
\text { Solution }\end{array}$ & $\begin{array}{l}\text { Zeroth-order } \\
\text { Solution }\end{array}$ & $\begin{array}{l}\text { First-order } \\
\text { Solution }\end{array}$ & $\begin{array}{l}\text { Exact } \\
\text { Solution }\end{array}$ \\
\hline$w(0)$ & -2.074985 & 12500 & 12500 & 12500 \\
$w(1)$ & -2.097810 & 100 & 100 & 100 \\
$w(2)$ & -2.120886 & 10 & 10 & 10 \\
$w(3)$ & -2.144216 & -2.144216 & -2.166506 & -0.443200 \\
$w(4)$ & -1.156802 & -1.156802 & -0.964915 & -0.361325 \\
$w(5)$ & -0.158527 & -0.158527 & 0.252251 & 0.690318 \\
$w(6)$ & 0.850729 & 0.850729 & 1.382978 & 1.733950 \\
$w(7)$ & 1.871087 & 1.871087 & 2.425218 & 2.682840 \\
$w(8)$ & 2.902669 & 2.902669 & 3.376886 & 3.537800 \\
$w(9)$ & 3.945598 & 3.945598 & 4.235865 & 4.307370 \\
$w(10)$ & 5 & 5 & 5 & 5 \\
\hline
\end{tabular}

(iii) Also note the very big boundary layer jumps at $k=0$ (from 12500 to -2.074985 ), at $k=1$ (from 100 to -2.09781 ) and at $k=2$ (from 10 to -2.120886 ), between the exact and degenerate solutions, indicating the nonuniform convergence and the effects of multi-time-scales (change in magnitudes of boundary layer jumps).

(iv) The first-order solution improves the zeroth-order solution and is much closer to the exact solution in the mean square sense.

\section{Main results and contributions of the paper}

(1) Development of a system model, in classical form, amenable to singular perturbation analysis for a class of linear time-invariant stable multi-time-scale digital control systems with several small parameters of widely different magnitudes.

(2) Transformations required, for boundary layer corrections that result in a unique solution, are provided.

(3) Perturbation methods are developed for possible initial and boundary value problems of the system considered.

\section{Conclusions}

So far singular perturbation methodology has been developed for mainly one- and two-parameter problems in digital control systems. The generalisation process of singular perturbation methodology, for any number of parameters in digital control systems represented in state space form, has already started $[5,6]$. The main aim 
of this paper is to present a generalised singular perturbation methodology for initial and boundary value problems of digital control systems represented in classical form. Accordingly singular perturbation methods have been developed for initial and boundary value problems of a stable linear time-invariant multi-parameter multi-time-scale digital control system with small parameters multiplying the lowest functions. Please note that the large number of corrections does not pose a problem due to the fact that all corrections need be evaluated for only a limited number of values of $k$ depending on the order of approximation, as shown in the illustrative example. The methods are given up to first-order approximation and can be easily extended to higher order approximations if required.

\section{Acknowledgements}

The author wishes to acknowledge his wife Nirmala for her encouragement throughout his research on singular perturbation methodology in digital control systems, Macquarie University for providing the necessary facilities and Dr B. D. Craven for a critical review.

\section{Appendix A.}

Consider a linear stable second-order difference equation [10]

$$
w(k+2)+d_{1} w(k+1)+d_{0} w(k)=0,
$$

with initial conditions $w(0)$ and $w(1)$. Here $d_{0}$ and $d_{1}$ are two small coefficients which are of different orders of magnitude such that $d_{0} \ll d_{1} \ll 1$. These two coefficients approach zero simultaneously in an interrelated manner.

The characteristic roots (eigenvalues) of (A.1) are

$$
z_{1,2}=d_{1}\left(-0.5 \pm 0.5 \sqrt{1-4 d_{0} / d_{1}^{2}}\right)
$$

The exact solution of (A.1) is

$$
w(k)=\left(\left(w(0) z_{2}-w(1)\right) z_{1}^{k}+\left(w(1)-w(0) z_{1}\right) z_{2}^{k}\right) /\left(z_{2}-z_{1}\right) .
$$

We obtain the trivial solutions demanded by (A.1) when we suppress the small coefficients for the following two limiting cases:

(1) $d_{0}=d_{1}^{2}$ as $d_{1} \rightarrow 0$; this is one-parameter problem with perturbation parameter $h=d_{1}$ and characteristic roots $z_{1,2}=h(-0.5 \pm 0.5 \sqrt{-3})$, a pair of complex conjugate roots representing fast modes. Now (A.1) may be written as

$$
w(k+2)+h w(k+1)+h^{2} w(k)=0 .
$$


(2) $\left(d_{0} / d_{1}^{2}\right) \rightarrow 0$ as $d_{1} \rightarrow 0^{+}$; this is a two-parameter problem with modified perturbation parameters $h_{1}=d_{1}$ and $h_{2}=d_{0} / d_{1}^{2}$. The characteristic roots

$$
z_{1,2}=h_{1}\left(-0.5 \pm 0.5 \sqrt{1-4 h_{2}}\right)
$$

are a pair of real roots representing fast and faster modes. Now (A.1) may be written in terms of these new parameters as

$$
w(k+2)+h_{1} w(k+1)+h_{1}^{2} h_{2} w(k)=0 .
$$

When we suppress $h_{1}$ and $h_{2}$ in (A.4), we get a trivial solution and the initial conditions $w(0)$ and $w(1)$ are lost in this degeneration process. These two initial conditions are to be recovered from the transformed equations. To find the corresponding transformations we approximate the roots of (A.3), assuming $\left|4 h_{2}\right|<1$, using the binomial expansions

$$
z_{1} \cong-h_{1} h_{2}\left(1+h_{2}\right) \text { and } z_{2} \cong-h_{1}\left(1-h_{2}\right) .
$$

By substituting (A.5) in (A.2), the zeroth-order solution of (A.2) may be obtained as

$$
w(k)=\left(-w(1) h_{1}^{k-1}+w(0)\left(h_{1} h_{2}\right)^{k}\right)(-1)^{k} .
$$

The form of (A.6) indicates that the transformations to be applied to (A.4) are

$$
w_{c}(k)=w(k) / h_{1}^{k-1} ; \quad w_{d}(k)=w(k) /\left(h_{1} h_{2}\right)^{k} .
$$

If we add a slow stable mode (which gives rise to a coefficient of $O(1)$, as the slow eigenvalues are of $O(1)$ in digital systems) to the system (A.1), case (1) becomes a two-time-scale system whereas case (2) becomes a three-time-scale system.

\section{References}

[1] F. Hoppensteadt, "Properties of solutions of ordinary differential equations with small parameters", Comm. Pure Appl. Math. 24 (1971) 807-840.

[2] A. Kailasa Rao and D. S. Naidu, "Singular perturbation method applied to the open-loop discrete optimal control problem", Opt. Control Appl. Methods 3 (1982) 121-131.

[3] H. Kando and T. Iwazumi, "Design of observers of stabilizing feedback controllers for singularly perturbed discrete systems", Proc. IEE D 132 (1985) 1-10.

[4] P. V. Kokotovic, R. E. O'Malley Jr and P. Sannuti, "Singular perturbations and order reduction", Automatica J. IFAC 12 (1976) 123-132.

[5] M. S. Krishnarayalu, "Singular perturbation methods for one-point, two-point and multi-point boundary value problems in multi-parameter digital control systems", J. EEE Australia 19 (1999) 97-110. 
[6] M. S. Krishnarayalu, "Singular perturbation analysis of a class of initial and boundary value problems in multi-parameter digital control systems", Control Theory Adv. Technology 10 (1994) 465-477.

[7] M. S. Krishnarayalu, "Singular perturbation method applied to the closed-loop discrete optimal control problem", Optimal Control Appl. Methods 11 (1990) 75-83.

[8] M. S. Krishnarayalu, "Singular perturbation method applied to the open-loop discrete optimal control problem with two small parameters", Int. J. Systems Sci. 20 (1989) 793-809.

[9] M. S. Krishnarayalu and D. S. Naidu, "Singular perturbation method for boundary value problems in two-parameter discrete control systems", Int. J. Systems Sci. 19 (1988) 2131-2143.

[10] M. S. Krishnarayalu and D. S. Naidu, "Singular perturbation method for initial value problems in two-parameter discrete control systems", Int. J. Systems Sci. 18 (1987) 2197-2208.

[11] D. S. Naidu, "Singular perturbations and time scales in control theory and applications: An overview", Dyn. Contin. Discrete Impuls. Syst. Ser. B Appl. Algorithms 9 (2002) 233-278.

[12] D. S. Naidu and A. Kailasa Rao, "Singular perturbation method for initial-value problems with inputs in discrete control systems", Int. J. Control 33 (1981) 953-965.

[13] D. S. Naidu and D. B. Price, "Singular perturbations and time scales in the design of digital flight control systems", NASA Technical paper 2844, 1988.

[14] R. E. O'Malley Jr, Introduction to singular perturbations (Academic Press, New York, 1974).

[15] P. K. Rajagopalan and D. S. Naidu, "A singular perturbation method for discrete control systems", Int. J. Control 32 (1980) 925-936.

[16] S. M. Roberts and J. S. Shipman, Two-point boundary value problems (Elsevier, New York, 1972).

[17] A. P. Sage and G. C. White III, Optimum system control (Prentice Hall, Englewood Cliffs, 1977).

[18] V. R. Saksena, J. O'Reilly and P. V. Kokotovic, "Singular perturbations and time-scale methods", Automatica J. IFAC 20 (1984) 273-293.

[19] G. P. Syrcos and P. Sannuti, "Singular perturbation modelling of continuous and discrete physical systems", Int. J. Control 37 (1983) 1007-1022. 